\title{
WC is better than BMI as a predictor of diabetes and pre diabetes in Hindu priests of India
}

\author{
Booloo Sharma1, Devajit Sarmah' \\ ${ }^{1}$ Department of Clinical Biochemistry, R D Gardi Medical College, Ujjain, India
}

\section{A B S T R A C T}

Background \& Objective: Over a period of time great emphasis has been made to prevent diabetes and its complications, and BMI have evolved into a reliable indicator for predicting the incidence of diabetes mellitus. But for a common man BMI is difficult to understand and calculate and there is a need for a more easily acceptable, understandable and measurable parameter as a predictor for diabetes risks. WC is one such parameter and the study tries to establish a correlation between (WC) and FBS with an aim to establish WC as a predictor of diabetes risks. Methods: From our ongoing diabetes screening study 220 Hindu priests were selected. This includes 55 newly diagnosed diabetes, 55 newly diagnosed pre-diabetes and 110 age matched controls. BMI, WC and FBS were calculated. A statistical correlation was established between BMI and FBS and between WC and FBS. The upper limit above which the risk association became statistically significant $(P<0.005)$ with pre-diabetes and diabetes were considered to be the cutoff for normal values. Results \& Conclusion: The WC was $31.77 \pm 2.63,35.82 \pm 1.04$ and $41.07 \pm 2.24$ inches in controls, pre-diabetes and diabetes respectively. A statistically significant difference $(P<0.005)$ for WC was found between controls and pre-diabetes and diabetes. Normal cut off for waist circumference among the Hindu priest is $<35$ inches or $<90$ centimeters. Also, statistically significant correlation was established between BMI and FBS and between WC and FBS however, correlation between WC and FBS is better than the former. WC more or less correlates with the pant/pyjama/trouser size, and almost everybody is aware of their pant size. Since WC is a more understandable term for the layman and which can be measured and tracked by the layman themselves, WC could be used as a better and more beneficial predictor of diabetes risk. Simply monitoring the pant size can make the layman understand when to ring the alarm and take medical attention or modify lifestyles.

Key words: Waist circumference (WC), Body mass Index (BMI), FBS, Better predictor
Access this article online

Website:

http://nepjol.info/index.php/AJMS

\section{INTRODUCTION}

Worldwide the prevalence of type-2 diabetes mellitus (T2DM) has been rising, and about $90 \%$ of the diabetic population are of T2DM. ${ }^{1}$ Of 371 million diabetic people worldwide 63 millions are Indian, i.e. every sixth diabetic is an Indian, as reported by the International Diabetic Federation (IDF) 2012 report., ${ }^{2,3}$

Diabetes has been associated with several risk factors. One of the most important risk factors is obesity. ${ }^{4,5}$ The association between increasing body mass index (BMI) and greater weight gain and risk of diabetes is most pronounced among Asians, suggesting that lower cut off BMI values are needed to identify Asians at a higher risk of diabetes. ${ }^{5-8}$ Contemporary research suggests that Indians may be genetically or phenotypically predisposed to store body fat viscerally. ${ }^{9,10}$ Central obesity is a common factor among Indians and so there is a probable indication that there is a preferential abdominal adiposity in Indians irrespective of the degree of general adiposity. ${ }^{10}$ Upper body obesity is associated with increased insulin resistance and diabetes. ${ }^{11}$ In recognition that visceral fat accumulation increases the risk of metabolic disease like diabetes, waist circumference 
(WC) has been used as an alternative surrogate measure of obesity. Though central obesity poses a greater risk for diabetes, it is the BMI which is more often used as a predictor of diabetes and pre-diabetes risks, but BMI gives an estimate of the generalized obesity rather than abdominal obesity. Moreover, BMI is a difficult term for the common man to understand. Calculation of BMI is also difficult for a common man. On the other hand WC which gives a perfect representation of the abdominal obesity is an easily understandable term, and can be a better predictor of diabetes and pre-diabetes. The study therefore tries to establish the association of WC with diabetes and pre-diabetes, and to verify if WC can be used as a better predictor of diabetes and pre-diabetes as compared to BMI.

\section{MATERIALS AND METHODS}

We selected 220 subjects from our ongoing population study where about 2000 Hindu priests of Central India are being screened for diabetes and its risk factors; starting from March 2013 and proposed to be over by November 2014. Of these 220 subjects 55 subjects were newly diagnosed case of diabetes and 55 were pre-diabetics and the remaining 110 subjects were taken as age matched controls. All known cases of diabetes were excluded from the study. As priesthood is practiced only my male Hindus, hence the subjects of our study were only males in the age group of 30 to 70 years. Clearance from Institutional ethical committee is obtained for conducting the study.

Sample for fasting sugar was estimated within 1 hour of collection by GOD/POD (Glucose oxidase/peroxidase) method using Vitros-250 dry chemistry analyser, manufactured by Ortho Clinical Division of Johnson and Johnson, USA. ${ }^{12}$

A general physical examinations and foot examination of the priests were done. Pulse and blood pressure (BP) were measured. Waist circumference, height and weight were measured and BMI were calculated. The height was measured from barefoot to the nearest $0.1 \mathrm{~cm}$ by a stadiometer. The body weight was recorded to the nearest $0.1 \mathrm{~kg}$ by using a calibrated weighing machine. The waist circumference was measured by using a measuring tape at the level which was midway between the lowest rib margin and the iliac crest.

\section{Definitions used}

Overweight was defined as body mass index (BMI) of $\geq 23 \mathrm{~kg} / \mathrm{m}^{2}$ and obesity as $\geq 25 \mathrm{~kg} / \mathrm{m}^{2} .{ }^{13}$

Statistical analysis was done by student-t test and Pearson's correlation manually using Microsoft excel sheet. The upper limit of WC above which, a significant association with pre-diabetes and diabetes occurred $(P<0.005)$ were considered to be the cutoff values of $\mathrm{WC}$ for pre-diabetes and diabetes, respectively.

Individuals with fasting plasma glucose (FPG) of $\geq 126 \mathrm{mg} / \mathrm{dl}$ or on medications for high blood sugar were considered to have diabetes mellitus and those with FPG between 110 to $125 \mathrm{mg} / \mathrm{dl}$ (Impaired fasting glucose) as prediabetics. ${ }^{1,14-16}$

\section{RESULT}

The study comprises of 55 diabetic, 55 pre-diabetics and 110 age matched controls with a mean age of $40 \pm 14.14$ years. The 55 diabetic and 55 pre-diabetics were newly diagnosed cases. The WC was $31.77 \pm 2.63$, $35.82 \pm 1.04$ and $41.07 \pm 2.24$ inches in controls, prediabetes and diabetes respectively. There is a statistically significant difference of WC between the control and diabetes $(\mathrm{P}<0.005)$; and between control and pre-diabetes $(\mathrm{P}<0.005)$. These findings are depicted by Table 1 . The study also established a correlation between FBS and BMI $(\mathrm{R}=0.539)$ and between FBS and WC $(\mathrm{R}=0.813)$ as shown by Figures 1 and 2 respectively. The study establishes that cut off WC for pre-diabetes is 35.82 inches or $90.98 \mathrm{~cm}$ and for diabetes it is 41.07 inches or $104.3 \mathrm{~cm}$, above which there is significant association with pre-diabetes and diabetes $(\mathrm{P}<0.005)$ respectively.

\section{DISCUSSION}

Diabetes is becoming an epidemic in India. ${ }^{1}$ Therefore there is an urgent need to generate easy, understandable and

\begin{tabular}{lccc}
\multicolumn{4}{l}{ Table 1: Waist circumference among controls } \\
\multicolumn{4}{l}{$(\boldsymbol{n}=\mathbf{1 1 0})$, pre-diabetes $(\boldsymbol{n}=\mathbf{5 5})$ and diabetes $(\boldsymbol{n}=55)$} \\
\hline Parameter & Controls (I) & Pre-diabetes (II) & Diabetes (III) \\
\hline WC (inches) & $31.77 \pm 2.63$ & $35.82 \pm 1.04$ & $41.07 \pm 2.24$ \\
P & Between & I \& II & I \& III \\
& & $<0.005$ & $<0.005$ \\
\hline
\end{tabular}

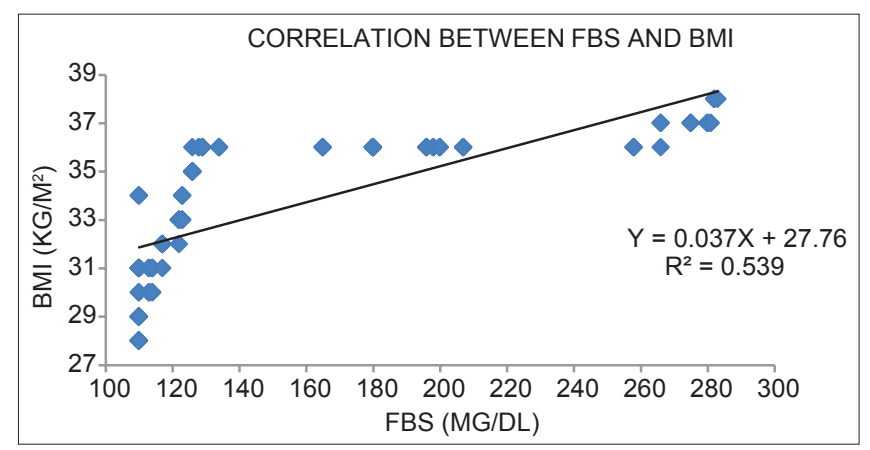

Figure 1: Correlation of fasting blood sugar (FBS) and body mass Index (BMI) among the pre-diabetes and diabetes 


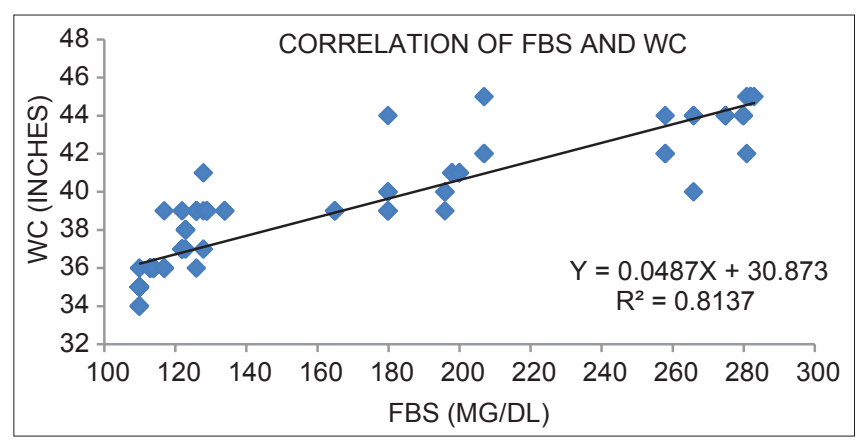

Figure 2: Correlation of fasting blood sugar (FBS) and waist circumference (WC) among the pre-diabetes and diabetes

effective ways for prevention of diabetes among Indians. BMI has been established as a predictor of diabetes risks among Indians, but it is difficult for a common man to calculate his or her BMI themselves. So there is a need for an effective and easily understandable parameter as a predictor for diabetes risk. Therefore the study tries to establish WC as one such predictor for diabetes risk. The study shows a statistically significant difference $(\mathrm{P}<0.005)$ in $\mathrm{WC}$ among normal controls and pre-diabetes, and WC among normal controls and diabetes. The study also shows that any $\mathrm{WC}$ above 35.82 inches $(35.82 \pm 1.04)$ or $90.98 \mathrm{~cm}$ is associated with pre-diabetes and WC above 41.07 inches (41.07 \pm 2.24$)$ or $104.3 \mathrm{~cm}$ is associated with diabetes. So, it can be established that normal cut off for waist circumference among the Hindu priest is $<35$ inches or $<90$ centimeters. Any WC above this is associated with diabetes risk.

We also found a good correlation between FBS and BMI with an $\mathrm{R}$ value of 0.539 , suggesting that increase in $\mathrm{BMI}$ is frequently associated with an abnormal FBS. Our findings are similar to other studies who showed a definite relation of BMI and diabetes mellitus. ${ }^{6,17}$ The important finding of our study is that we found a better correlation between WC and FBS $(\mathrm{R}=0.813)$, than between BMI and FBS $(\mathrm{R}=0.539)$.

Most patients do not understand the importance of body mass index or BMI which involves two parameters - weight and height. However waist circumference is an easily understandable term, can be measured by patient themselves, and they can regularly monitor it. A person may not know their BMI but they know their pant (trouser/ payzama) size, which more or less correlates with the waist circumference. ${ }^{5}$ People can be motivated to keep a track of their pant size, and whenever it exceed 35 inches $(90 \mathrm{~cm})$ they take it as an alarm and modify their lifestyles and get their blood sugar tested.

We feel that assigning risk for diabetes based on pant size ( $\approx$ waist circumference) can make the layman understand the effect of obesity, and they can very well monitor their waist circumference. This can be more beneficial than $\mathrm{BMI}$ cut off as a risk factor. Interestingly the study also shows that BMI and WC are very well correlated with FBS, correlation of later is however slightly better. This can also be helpful for health education provider who can make the layman understand to track their WC rather than track their BMI. Importantly abdominal obesity is more associated with insulin resistance than overall obesity, and WC is the most affected parameter by abdominal obesity. So in this context the usefulness of WC as a predictive marker exceeds that of BMI.

Our study was conducted in Hindu priest community and though we feel that the findings may be same in the general population, a large scale study on the general population is needed to support these findings. Further we have not taken females into consideration as they do not perform the duty of a priest, and a similar study on women is therefore needed. Thus these are the demerits of our study.

\section{CONCLUSION}

The study establishes that any waist circumference above 35 inches or $90 \mathrm{~cm}$ is frequently associated with diabetes risk among the Hindu priests. We finally conclude that though BMI was considered as a more accurate predictor of diabetes risk, the layman finds it difficult to understand and measure BMI. But pant or trouser size which often correlates with WC is known to all, and therefore it can be a better predictor for diabetes risk among the masses. Moreover people can be easily motivated to keep a track of their pant size, and thus WC can be a better parameter than BMI as a predictor for diabetes risks.

\section{ACKNOWLEDGEMENT}

We are thankful to the Ortho Clinical Division of Johnson and Johnson, India for funding the population study for screening the Hindu priest community for diabetes, from which the study subjects were selected.

\section{REFERENCES}

1. Sarmah D and Sharma B. Importance and Status of HBA1C in T2DM and its Indian Perspective. Asian Journal of Biomedical and Pharmaceutical Sciences 2012; 2(12):1-10.

2. International diabetic Federation Annual report (2012) Available at www.idf.co.in. Assessed online August 2013.

3. Sharma B, Sarmah D and Sonker P. Effect of different anticoagulants on HBA1C estimation and its stability. J Lab Physicians 2013; 5:143-144.

4. Sarmah D, and Sharma B. A correlation of Uric acid and lipid profile. Asian Journal of Medical Sciences (AJMS) 2013; 4(2): 8-14. 
5. Sarmah D and Sharma B. Diabetes and HbA1c: An Indian perspective. Germany: Lap Lambert Academic Publishing, 2014, pp 19-39.

6. Schienkiewitz A, Schulze MB, Hoffmann K, Kroke A and Boeing $\mathrm{H}$. Body mass index history and risk of type 2 diabetes: results from the European Prospective Investigation into Cancer and Nutrition (EPIC)- Potsdam Study. American Journal of Clinical Nutrition 2006, 84(2):427-433.

7. Sauvaget C, Ramadas K, Thomas G, Vinoda J, Thara S and Sankaranarayanan R. Body mass index, weight change and mortality risk in a prospective study in India. International Journal of Epidemiology 2008, 37:990-1004.

8. Goel K, Misra A, Vikram NK, Poddar P and Gupta N. Subcutaneous abdominal adipose tissue is associated with the metabolic syndrome in Asian Indians independent of intraabdominal and total body fat. Heart 2010; 96(8):579-583.

9. Mohan V, Deepa M, Farooq S, Narayan KM, Datta M and Deepa R. Anthropometric cut points for identification of cardiometabolic risk factors in an urban Asian Indian population. Metabolism Clinical and Experimental 2007, 56:961-968.

10. Ramachandran A, Snehalatha $C$ and Viswanathan V. Burden of type 2 diabetes and its complications - The Indian scenario. Current Science 2002, 83:1471-1476.
11. Lundgren $\mathrm{H}$, Bengtsson $\mathrm{C}$, Blohme $\mathrm{G}$, Lapidus $\mathrm{L}$ and Sjöström $\mathrm{L}$. Adiposity and adipose tissue distribution in relation to incidence of diabetes in women: results from a prospective population study in Gothenburg, Sweden. Int J Obes 1989;13(4):413-423.

12. Trinder P. Determination of blood glucose using an oxidaseperoxidase system with a non-carcinogenic chromogen. J. Clin. Pathol 1969; 22 (2): 158-161.

13. Snehalatha $C$, Viswanathan $V$ and Ramachandran $A$. Cut off Values for Normal Anthropometric Variables in Asian Indian Adults. Diabetes Care 2003; 26:1380-1384.

14. Alberti KG and Zimmet PZ. Definition, diagnosis and classification of diabetes mellitus and its complications.Part 1: Diagnosis and classification of diabetes mellitus provisional report of a WHO consultation. Diabet Med 1998; 15:539-553.

15. American Diabetes Association Diagnosis and classification of diabetes mellitus. Diabetes Care 2012; 35(1):S64-S71.

16. Sarmah D and Sharma B. Can Hba1c Prove Helpful in Diagnosis of Type 2 DM in India? Scholars Journals of Applied Medical Sciences 2013; 1(6):789-792.

17. Sauvaget C, Ramadas K, Thomas G, Vinoda J, Thara S and Sankaranarayanan R. Body mass index, weight change and mortality risk in a prospective study in India. International Journal of Epidemiology 2008; 37:990-1004.

\section{Authors Contribution:}

BS and DS - contributed equally towards study design; data collection, analysis and interpretation; and writing of manuscript.

Source of Support: Nil, Conflict of Interest: None declared. 